\title{
SYNTHESIS OF GRAFITE FROM AMAZONIAN RESIDUAL BIOMASS
}

\section{Jeorge Lucas dos Santos Monteiro', Thalysson Clementino da Cruz ${ }^{2}$, Anderson de Oliveira Castro ${ }^{3}$}

1,2,3 Centro Universitário do Norte - UNINORTE - Laureate Universites, Brasil, Rua Leonardo Malcher, 715 - Centro, Manaus/AM.

Email: jeorgelucas13@gmail.com, thalyson.eng@outlook.com, anderson.castro@ uninorte.com.br

Received: June $15^{\text {th }}, 2019$

Accepted: June 19th 2019

Published: September $30^{\text {th }}$, 2019

Copyright $@ 2016$ by authors and Institute of Technology Galileo of Amazon (ITEGAM).

This work is licensed under the Creative Commons Attribution International License (CC BY 4.0). https://creativecommons.org/lice nses/by/4.0/

\section{ABSTRACT}

The Amazon is the largest tropical forest in the world, with the richest biodiversity on the planet, producing a range of typical and exotic fruits, as well as housing native trees that make up its biome. The Amazon is the largest tropical forest in the world, with the richest biodiversity on the planet, producing a range of typical and exotic fruits, as well as housing native trees that make up its biome. The residual biomass ends up interfering negatively in the environment, because it is discarded like garbage, bringing losses that go from the clogging of sewers to the proliferation of diseases. In a visionary perspective, many researchers are working on the exploration of these biomasses for their use in the science and engineering of new materials, thus collaborating with the minimization of these wastes in the environment, using the so-called sustainable technological development. For this work the residual biomass from the açaí stone was chosen, since only $15 \%$ of the fruit is harvested, being discarded as reject $85 \%$ in the urban environment. Thus, as the biomass consists of three main fractions, cellulose, hemicellulose and lignin, which contains abundant hydroxyl and carbonyl groups, these characteristics allow the maceration of açaí stone to coordinate with several metallic ions, which can be used for the synthesis of carbon materials such as graphene and hence graphite, a material used to produce supercapacitor electrodes (EDLCs), which use carbon as an active material for the storage of electricity with high power density and life cycle. In this context, the present work aims to perform the graphite synthesis from the Amazonian residual biomass (açaí stone).

Keywords: Synthesis. Graphite. Residual biomass. Amazon. Supercapacitor.

\section{SÍNTESE DE GRAFITE A PARTIR DE BIOMASSA RESIDUAL AMAZÔNICA}

\section{RESUMO}

A Amazônia é a maior floresta tropical que existe no mundo, com a mais rica biodiversidade do planeta, produzindo uma gama de frutos típicos e exóticos, além de abrigar árvores nativas que compõem seu bioma. A biomassa residual acaba por interferir negativamente no meio ambiente, pois é descartada como lixo, trazendo prejuízos que vão desde o entupimento de esgotos até a proliferação de doenças. Em um panorama visionário, muitos pesquisadores estão com a missão de explorar essas biomassas para fins de sua utilização em ciência e engenharia de novos materiais, colaborando assim com a minimização desses rejeitos no ambiente, empregando o chamado desenvolvimento tecnológico sustentável. Para este trabalho escolheu-se a biomassa residual proveniente do caroço do açaí, pois são aproveitados somente $15 \%$ do fruto, sendo descartado como rejeito $85 \%$ no ambiente urbano. Dessa forma, como a biomassa é composta por três frações principais, celulose, hemicelulose e lignina, que contém abundantes grupos de hidroxila e carbonila, essas características permitem que o macerado de caroço de açaí coordene com vários íons metálicos, o que pode ser usado para a síntese de materiais de carbono, tais como o grafeno e consequentemente o grafite, material utilizado para a produção de eletrodos supercapacitores (EDLCs), que utilizam o carbono como material ativo para armazenamento de energia elétrica com alto poder de densidade e ciclo de vida. Nesse contexto, o presente trabalho tem como objetivo realizar a síntese de grafite a partir da biomassa residual amazônica (caroço de açaí).

Palavras-chave: Síntese. Grafite. Biomassa residual. Amazônia. Capacitores. 


\section{INTRODUÇÃO}

A Amazônia é a maior floresta tropical que existe no mundo, com a mais rica biodiversidade do planeta. Entre as variedades de animais e plantas, a região também produz uma grande diversidade de frutas típicas e exóticas, além de abrigar árvores nativas que compõem sua biomassa [1].

Nesse relance de frutos multivariados, verifica-se que a quantidade de insumos e resíduos produzidos a partir do seu consumo e utilização aumenta de maneira exponencial ao passar dos anos. Essa relevante concentração de biomassa residual amazônica acaba se transformando em lixo, pois não são descartadas de maneira correta e na maioria dos casos agrava o meio ambiente trazendo prejuízos que vão desde o entupimento de esgotos até a proliferação de doenças.

Uma biomassa residual amazônica em expansão é o caroço de açaí, onde o seu segmento produtivo é uma cultura consolidada no Estado do Pará, sendo este o maior produtor brasileiro, perfazendo $55 \%$ da produção nacional (111.073 toneladas, em março de 2015) e o maior consumidor possuindo um mercado de consumo tradicional e enraizado na sua própria região de origem [2].

Existem diversas aplicabilidades para esses resíduos, como por exemplo, na forma de amêndoa (panificação, fabricação de ração e fitoterapia), fibras (isolamento acústico, indústria automobilística, entre outros) e microfibras (indústria cosmética) [1].

O potencial desta semente faz jus á importância do fruto para o Estado, variando entre a produção de energia e o tratamento para câncer, passando até mesmo pela aplicação do rejeito para fins de fabricação de móveis. Vale ressaltar que estes dados levam em conta apenas o consumo da capital e municípios circunvizinhos. $\mathrm{O}$ consumo do estado inteiro pode elevar estes números às milhares de toneladas ao ano.

Além do destino final dado a variedade de biomassa residual existente na região amazônica, como é o caso do caroço de açaí, existem outras problemáticas que precisam ser solucionadas para a melhoria das condições de vida do planeta, em se tratando de energia, meio ambiente e clima.

Na sociedade atual, onde os modos de consumo de energia se intensificam a cada dia, a busca por alternativas eficazes de produção, distribuição e diversificação de energia tornam-se essenciais diante da dependência dos recursos energéticos [3].

Ao passo que as mudanças climáticas se tornaram mais intensas e o esgotamento dos combustíveis fósseis mais eminentes, as fontes de energia renováveis ganharam impulso como fontes viáveis de energia mais econômicas e favoráveis ao meio ambiente. Entretanto, a maioria das fontes de energia renováveis são inconstantes e intermitentes, sendo, portanto, necessário realizar estratégias e estudos voltados para os sistemas de armazenamento.

Entre os diversos sistemas de armazenamento, os supercapacitores são os proponentes mais promissores para o armazenamento de energia não somente nas energias renováveis, mas também em veículos híbridos e dispositivos portáteis devido à sua alta densidade de potência. Os eletrodos supercapacitores são geralmente feitos de carbono derivado da biomassa [4].

A biomassa é composta por três frações principais, celulose, hemicelulose e lignina, que contém abundantes grupos de hidroxila e carbonila, sendo que essas características permitem quer o macerado de caroço de açaí coordenem com vários íons metálicos, o que pode ser usado para a síntese de materiais de carbono, tais como o grafeno.

O grafeno é um material bidimensional formado por átomos de carbono com uma estrutura cristalina hexagonal, cujas propriedades mecânicas e eletrônicas têm atraído interesse nas áreas tecnológica e de estudo fundamental. As propriedades físicas do grafeno são semelhantes àquelas dos nanotubos de carbono, porém possui uma superfície especifica maior $(\sim 2620 \mathrm{~m} 2 / \mathrm{g}$ teórico) [5].

Por apresentar propriedades diferenciadas, o grafeno tem atraído considerável interesse tecnológico. Os processos de preparação envolvem diferentes metodologias, que se baseiam na produção do óxido de grafite (GO) e sua posterior redução, com a formação do grafeno [6].

Nesse contexto, a síntese de grafite passou a ter uma visão mais ampliada para fins de armazenamento de energia pela biomassa residual, sendo que nos dias atuais, as baterias secundárias são os sistemas de armazenamento mais utilizados.

As baterias armazenam energia quimicamente, tanto nos eletrólitos quanto nos eletrodos que acabam sofrendo alterações físico-químicas durante o carregamento e a descarga, levando assim a baixa ciclosidade e baixa vida útil do material [7].

Assim suas aplicabilidades em armazenamento de energia são limitadas. Ao contrário das baterias, os supercondensadores possuem alta densidade de potência e estabilidade de ciclagem. Porém, sua utilidade no armazenamento de energia é limitada por sua baixa densidade de energia, resistência de série efetiva relativamente alta e alto custo [2,3].

Já os supercapacitores apresentam uma densidade de energia inferior à das baterias de ácido chumbo e íon de lítio. No entanto, eles têm maior densidade de potência e exibem ciclos de vida longos, alta capacidade de ciclagem e baixas preocupações de segurança em comparação com baterias secundárias, atraindo a atenção como novo sistema de armazenamento de energia [8].

Á priori, os supercondensadores armazenam energia com base em dois comportamentos capacitivos: capacitância elétrica de camada dupla (EDL) e pseudocapacidade. Enquanto o primeiro é devido à interação eletrostática, a pseudocapitância é devido ao fenômeno farádico que envolve reações eletroquímicas rápidas e reversíveis.

$\mathrm{Na}$ pseudocapitância, as reações de redox estão entre o eletrólito e os materiais dos eletrodos. Os produtos das reações de redox são, portanto, elétrons que são então transferidos através de interfaces eletrodo / eletrólito [6,7,8].

O EDL tem a vantagem de que a cinética eletroquímica não está limitada pela resistência à polarização e nenhum inchaço dos materiais ativos é experimentado. A energia é armazenada em dois eletrodos porosos com coletor de corrente em cada eletrodo; a energia armazenada é liberada quando as cargas acumuladas na descarga do eletrodo.

O número de cargas armazenadas no eletrodo é proporcional à superfície do eletrodo e a energia armazenada é proporcional à quantidade de carga armazenada. Portanto, os materiais com alta área de superfície são considerados bons candidatos para eletrodo supercapacitor.

A melhoria do desempenho dos supercondutores pode ser atribuída por meio da descoberta de novos materiais de eletrodos, pela compreensão do comportamento dos íons em poros pequenos e pela concepção do sistema híbrido combinando eletrodos farádico e capacitivos. É de extrema importância transformar o macerado de caroço de açaí em materiais de carbono com estruturas específicas devido às ricas espécies carbonáceas que estão contidas nelas, dando uma maior aplicabilidade a essa biomassa residual proveniente da região amazônica.

Tais propriedades permitem que a biomassa residual do açaí seja uma matéria-prima eficaz para a sintetização de materiais de carbono com estruturas específicas para certos condensadores de dupla camada eletroquímicos, mais conhecidos como 
supercapacitores (EDLCs), que utilizam o carbono como material ativo, uma vez que os supercapacitores são uma classe de dispositivo para armazenamento de energia elétrica com alto poder de densidade, ciclo de vida longo, captura e armazenamento rápidos de energia [9].

Há pouco tempo, nanomateriais de carbono 2D com estruturas porosas estão atraindo enorme atenção na área de EDLCs devido a eles possuírem excelente propriedade de transporte eletrônico, como a do grafeno [101].

Diversos trabalhos $[3,4,5,6,7,9]$ demonstram que o revestimento de carbono mesoporoso no grafeno e em grafite apresenta-se com excelência capacitância eletroquímica e ciclismo de alta estabilidade.

Devido a esse grande êxito das pesquisas houve uma maior inspiração para a sintetização de nanopartículas de grafite porosa (NPGP) como eletrodos para supercapacitores.

Nesse caso, a fim de minimizar a poluição ambiental e a escassez de recursos, é importante desenvolver um método econômico para a preparação de grafite para uso em EDLCs que são derivados da biomassa, tais como macerado de caroço de açaí.

$\mathrm{Na}$ síntese de grafite, o macerado de caroço de açaí é primeiramente coordenado com $[\mathrm{Fe}(\mathrm{CN}) 6] 4$ e em seguida após a sua carbonização e remoção do catalisador, o NPGP é obtido.

A estrutura única do NPGP resultante permite um bom desempenho em supercapacitores (EDLs). Um dos fatores positivos dessa sintetização é a formação de uma estrutura altamente porosa e benéfica para dar acessibilidade eletrolítica e rápida difusão no transporte de íons, fornecendo uma curta distância de difusão iónica [5,7].

Outro fator vantajoso é a excelente condutividade do plano 2D em nanopartículas de grafite, utilizada para facilitar $\mathrm{o}$ transporte rápido de elétrons durante processos eletroquímicos. Finalmente, independente do caminho que o NPGP está conectado, isto é, em paralelo ou em série, o NPGP, que atua como minicorrente de colecionadores, podem formar uma rede condutora no eletrodo $[6,7,8]$.

A nanopartícula de grafite porosa sintetizada pode ser usada como eletrodos para avançar supercondicionadores com excelentes performances capacitivas. Nesta pesquisa utilizaremos o caroço de açaí como biomassa residual amazônica para fins de sintetizar o grafite, levando em consideração as suas condições nanotecnológicas como supercapacitores.

Dessa forma, esta pesquisa tem como objetivo analisar uma amostra de grafite, proveniente do caroço do açaí, utilizando-se da técnica de difração de raios-X, usando o equipamento do Laboratório de Materiais (LabMat) da Universidade Federal do Amazonas (UFAM).

\section{METODOLOGIA}

A biomassa residual amazônica a ser estudada nesta pesquisa é o caroço de açaí (Figura 1), fruto proveniente da palmeira Euterpe olerace (conhecida como açaizeiro), considerada como a palmeira de maior importância econômica, social e cultural da região norte do Brasil, onde o Estado do Pará se destaca como o maior produtor e consumidor. Sendo assim, os caroços de açaí serão coletados nas ruas da cidade de Belém, no Pará.

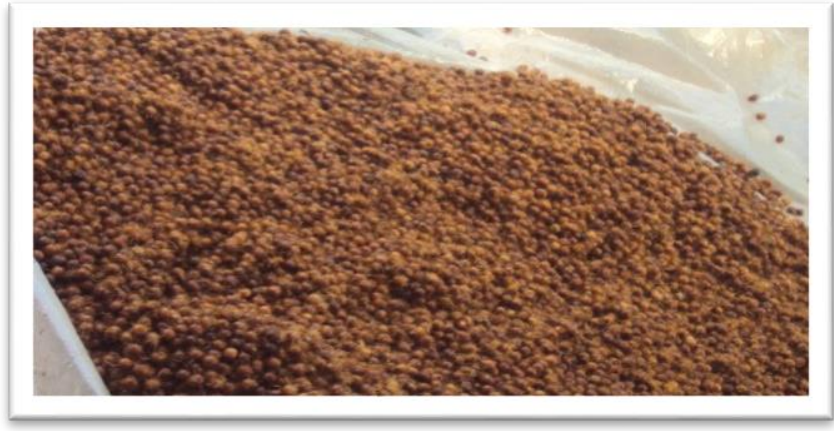

Figura 1 - Biomassa residual Amazônica. Fonte: Autores, (2019).

Após serem coletadas, as amostras de caroço de açaí foram secadas em estufa por $72 \mathrm{~h}$, a uma temperatura de $100^{\circ} \mathrm{C}$ (Figura 2).

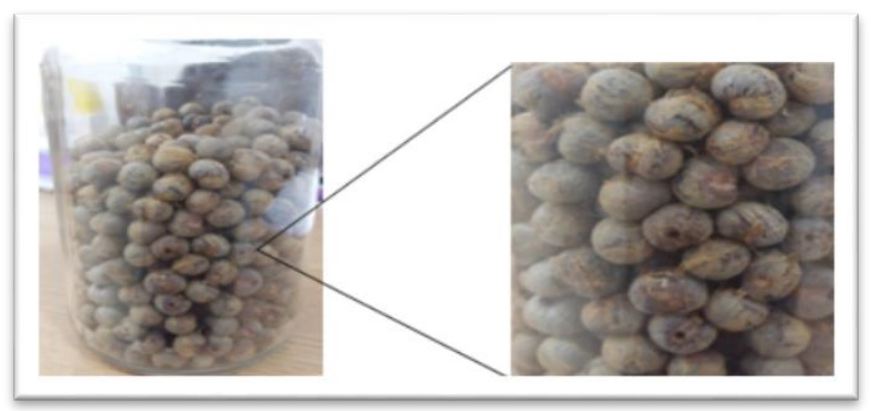

Figura 2 - Caroços de açaí.

Fonte: Autores, (2019).

A posteriori, os caroços foram triturados para melhor condicionar os testes de caracterização do material.

Em seguida foi pesado, em uma balança analítica 2,6 $\mathrm{g}$ de biomassa residual e imergida em $180 \mathrm{~mL}$ de soluções aquosas de $\mathrm{K} 4\left[\mathrm{Fe}(\mathrm{CN})_{6}\right], \quad \mathrm{COCl}_{2}, \quad \mathrm{ZnCl}_{2}, \quad \mathrm{LiCl}, \quad \mathrm{Fe}_{2}\left(\mathrm{SO}_{4}\right)_{3} \quad$ e FeSO4. $\left(\mathrm{NH}_{4}\right)_{2} \mathrm{SO}_{4} \cdot 6 \mathrm{H}_{2} \mathrm{O}, \mathrm{FeCl}_{3}$, todas a uma concentração de $0,1 \mathrm{M}$, durante 4 horas, à uma temperatura de $35^{\circ} \mathrm{C}$, com a finalidade de proporcionar a formação do compósito BIOMASSAÍON METÁLICO.

$\mathrm{Na}$ sequência metodológica, o sólido foi recolhido e conduzido ao forno para procedimento pirolítico, durante 2 horas, nas temperaturas de $900^{\circ} \mathrm{C}$ e $1100^{\circ} \mathrm{C}$, com uma taxa de aquecimento constante de $5^{\circ} \mathrm{C} /$ minuto em nitrogênio atmosférico.

Logo após essa etapa, a amostra foi tratada com $120 \mathrm{~mL}$ de ácido clorídrico $(\mathrm{HCl}) 1,0 \mathrm{M}$ à $80^{\circ} \mathrm{C}$, durante 6 horas.

O compósito obtido foi lavado com água destilada até ser neutralizado e seco em estufa, à $80^{\circ} \mathrm{C}$.

Após a preparação do material analítico a ser estudo, realizou-se a caracterização do grafite por meio da DRX.

A parte experimental deste trabalho foi realizada no Laboratório de Materiais - (LabMat) da Universidade Federal do Amazonas, teve como objetivo apresentar características sobre funcionamento do equipamento de difração de raio $\mathrm{X}$, bem como coleta de dados necessário à análise da grafite.

Após a preparação do primeiro material a ser analisado o porta-amostras foi fixado no equipamento de difração de marca Panalytical e modelo: Empyrean (Tabela 1) e analisado de acordo com parâmetros presentes na tabela abaixo. 
Tabela 1: Parâmetros para análise.

\begin{tabular}{|c|c|c|c|}
\hline $\begin{array}{c}\text { Passo } \\
\text { (graus) }\end{array}$ & $\begin{array}{c}\text { Radiação } \\
\text { Incidente } \\
\mathrm{k} \alpha(\mathrm{A})\end{array}$ & $\begin{array}{l}\text { Faixa de } \\
\text { Leitura } 2 \Theta \\
\text { (graus) }\end{array}$ & $\begin{array}{c}\text { Tempo } \\
\text { aproximado } \\
\text { de Medida }\end{array}$ \\
\hline 0,02 & $\mathrm{Cu}-0,15418$ & $10-100$ & $10 \mathrm{~min}$ \\
\hline
\end{tabular}

Fonte: Autores, (2019).

Após o término das análises de difração de raio-x, três arquivos na extensão xy, foram gerados e encaminhados por e-mail para que pudéssemos utilizar na geração dos difratogramas correspondentes a cada amostra.

$\mathrm{Na}$ base de dados ICSD [1,2,3] foi feita a escolha de um arquivo de alta qualidade cartão 51688 com as características apresentadas na (Figura 3) descrita a seguir:

\begin{tabular}{|c|c|c|c|c|}
\hline \multicolumn{4}{|l|}{ Summay } & \multirow{2}{*}{ 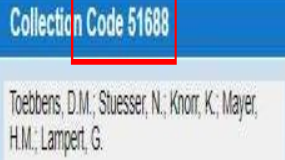 } \\
\hline Stuctiomila & \multicolumn{2}{|l|}{ S } & Author & \\
\hline Space Group & \multicolumn{2}{|l|}{ Fo.3ms 2277} & \multirow{3}{*}{ Tileo of Aficte } & \\
\hline Unitcell & \multicolumn{2}{|c|}{ 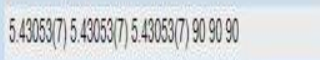 } & & \multirow{2}{*}{ 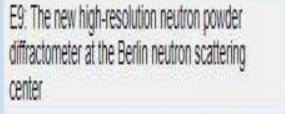 } \\
\hline Cell Voune & $100.15 A^{2}$ & Fomua Unis per Cell 8 & & \\
\hline Temperatue & rom temperalue & essure ammospheic & Reiefence & 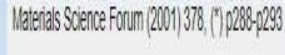 \\
\hline PDF-Nunbers & 01.070 .5680 & R. Halle & Warings \& Comments & 1 Warings / 1 Commells \\
\hline Remarín & & 3 Higho & & \\
\hline
\end{tabular}

Figura 3 - Cartão 51688 - ICSD.

Fonte: Autores, (2019).

O programa Mercury 3.9 foi baixado gratuitamente do site:https://www.ccdc.cam.ac.uk/Community/csdcommunity/free mercury/, nele foram parametrizados pontos importantes do ICSD51688 para que fossem comparados com os dados do experimento, conforme vermos nos pontos a seguir. Identificação da célula unitária da grafite (Figura 4).

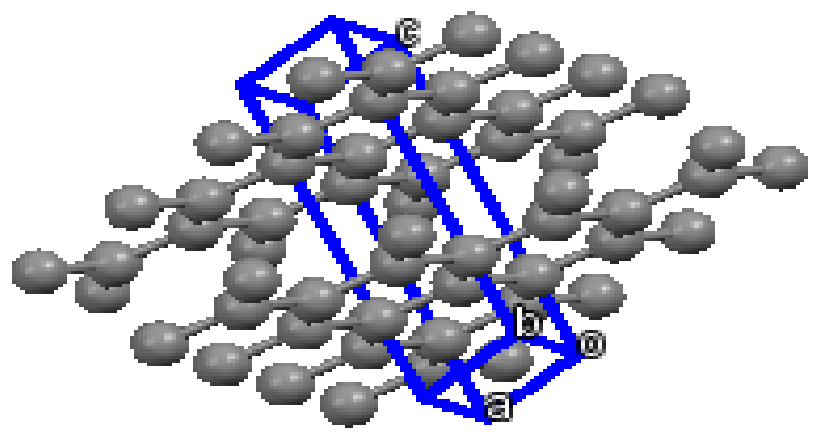

Figura 4 - Célula unitária do grafite no Mercury. Fonte: Autores, (2019).

A seleção do comprimento de onda do cobre foi estabelecida na faixa de varredura entre $10^{\circ}-100^{\circ}$ e passo de $0,02^{\circ}$ conforme aplicado durante o experimento, bem como a identificação dos índices de miller (Figura 5), além da geração de arquivo na extensão .xy e para posterior plotagem no OriginPro 2016.

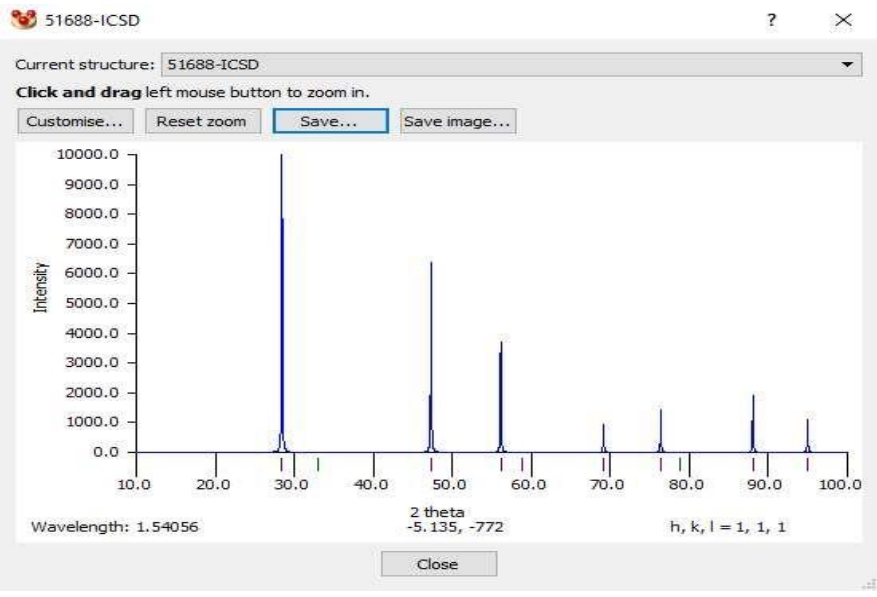

Figura 5 - Índices de Miller.

Fonte: Autores, (2019).

\section{RESULTADOS E DISCUSSÕES}

Por meio da efetivação da proposta de melhoria no setup de abastecimento de linha em uma empresa de motocicletas da Cidade de Manaus

Em conformidade com a natureza da técnica de DRX, os dados experimentais obtidos foram utilizados para confirmar as seguintes informações (DU et al., 2004):

Composição de fase de um material, incluindo análises qualitativas e quantitativas, juntamente com buscas de vários bancos de dados;

Índices de reflexões de Bragg, intensidades integradas observadas e parâmetros de rede precisos;

Distribuição das características da célula comum, a estrutura cristalina, quer para que o material tenha um dos já conhecidos tipos de estruturas cristalinas, ou para resolvê-lo dos primeiros princípios;

Detalhes estruturais precisos, incluindo posições de equilíbrio de átomos na célula unitária, deslocamento atômico individual e Parâmetros populacionais empregando o método Rietveld;

Várias características estruturais microscópicas da amostra.

Dessa forma, pôde se avaliar o comportamento da grafite em cada resposta do DRX, levando em consideração a grafite pura e com 12 horas de moagem.

No gráfico (Figura 6), referente a grafite pura, percebe-se a presença de um pico de difração (002) de intensidade elevada a $2 \theta$ de $26,56^{\circ}$ corresponde ao espaçamento basal de $0,335 \mathrm{~nm}$ característico do grafite puro.

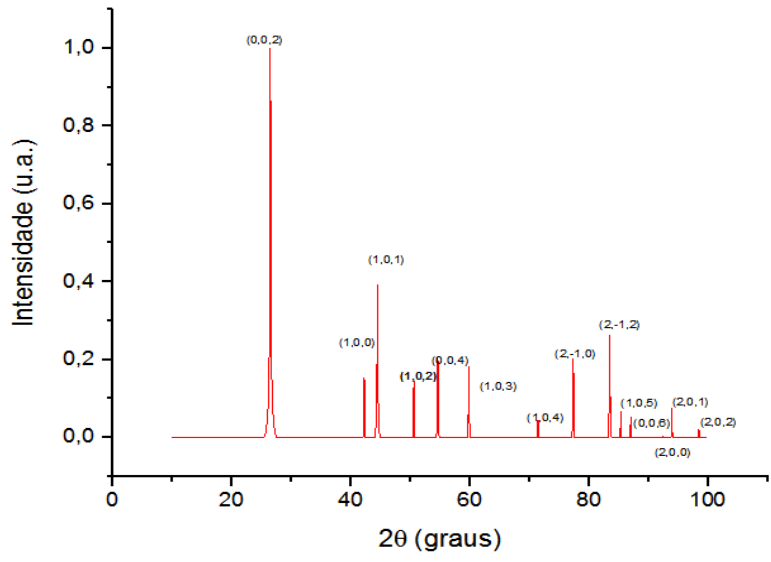

Figura 6 - Índices de Miller.

Fonte: Autores, (2019). 
Este pico de difração, geralmente é um indicador que tem como finalidade avaliar o grau de oxidação da grafite. Isso pode ser verificado comparando-se o espectro de DRX obtido para a grafite e para o óxido de grafite, onde a ausência deste pico de difração (002) e o aparecimento de um pico a $2 \theta$ de $10^{\circ}$ no óxido de grafite implica em uma completa oxidação do grafite.

A caracterização por difração de raios $\mathrm{X}$ também forneceu dados que permitiram o cálculo dos parâmetros de rede das amostras de grafite resultantes das duas sínteses em estudo, utilizando-se a equação de $\operatorname{Bragg}(2 \mathrm{~d} \sin \Theta=\lambda)$ onde $\mathrm{d}$ é à distância interplanar no cristal, $\Theta$ é o ângulo entre o vetor de onda incidente e os planos cristalinos da amostra e $\lambda(1,54 \AA$ Á) é o comprimento de onda da radiação incidente.

$\mathrm{O}$ trecho dos difratogramas de raios $\mathrm{X}$ aqui mencionados abrangeu o ângulo $2 \theta$ entre 10 e $80^{\circ}$, onde aplicando a Lei de Bragg foi possível identificar um pico de difração em $2 \theta$ igual a $10^{\circ}$, o qual pode ser observado nos espectros DRX apresentados nos gráficos 1 e 2 .

A grafite por apresenta uma estrutura do tipo lamelar, é formada por planos de átomos de carbono arranjados de forma hexagonal (camadas de grafeno) em hibridização do tipo sp2. Logo, estas camadas são ligadas entre si, principalmente, por forças de interação fraca do tipo Van der Walls, permitindo deste modo a intercalação de grandes quantidades de espécies químicas (moléculas e íons) no espaçamento entre os planos, uma vez que a distribuição eletrônica dos planos é facilmente rearranjada.

Nesse fundamento são possíveis a formação de duas estruturas plausíveis para a grafite, dependendo da distribuição dos planos de átomos de carbono: estrutura hexagonal ou romboédrica.

As grafitas naturais apresentam-se quase sempre na fase hexagonal adquirindo fase romboédrica durante o processo de pulverização do mineral, apresentando uma condução elétrica anisotrópica, ou seja, a condutividade ocorre de maneira diferenciada na direção dos planos basais e na direção perpendicular aos planos, sendo muito maior (da ordem de $3 \mathrm{x}$ ) a condutividade no plano basal, do que no sentido perpendicular dos planos.

No gráfico (Figura 7), percebeu-se a presença de um pico mais intenso e estreito para a amostra de grafite com 12 horas de moagem.

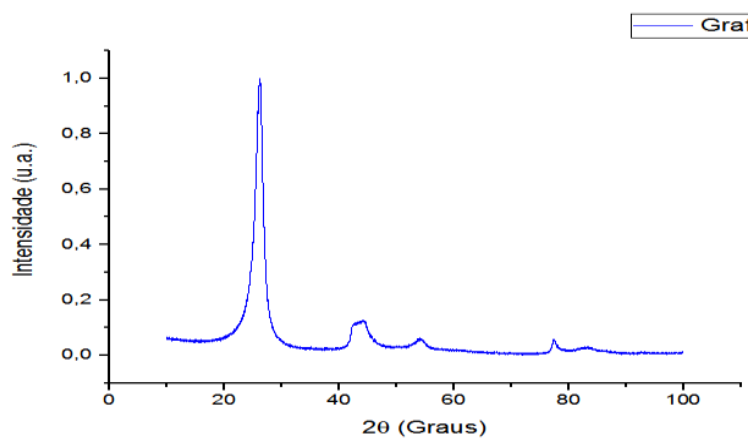

Figura 7 - Gráfico com 12 horas de moagem. Fonte: Autores, (2019).

Esta diferença na intensidade e largura a meia altura do pico de Difração de Raios-X das amostras de grafite pode estar relacionada com o tamanho dos cristalitos. O tamanho médio dos cristalitos (L) foi calculado utilizando a fórmula de Scherrer, apresentada a seguir:

$$
\mathrm{L}=\mathrm{K} \lambda / \beta \cos \theta
$$

Onde, $\beta$ é a largura a meia altura do pico obtido pelo ajuste do pico, $\lambda$ é o comprimento de onda da radiação incidente (1,54 Á) que depende do equipamento utilizado, e o valor de $\mathrm{K}$ depende do material a ser analisado, que para o carbono é de 0,90 , onde verifica-se que o existe uma maior qualidade estrutural para o grafite com $12 \mathrm{~h}$ de moagem. Realizando a plotagem entre os três parâmetros amostrais percebemos o aparecimento descontínuo do deslocamento dos picos no gráfico a seguir (Figura 8).

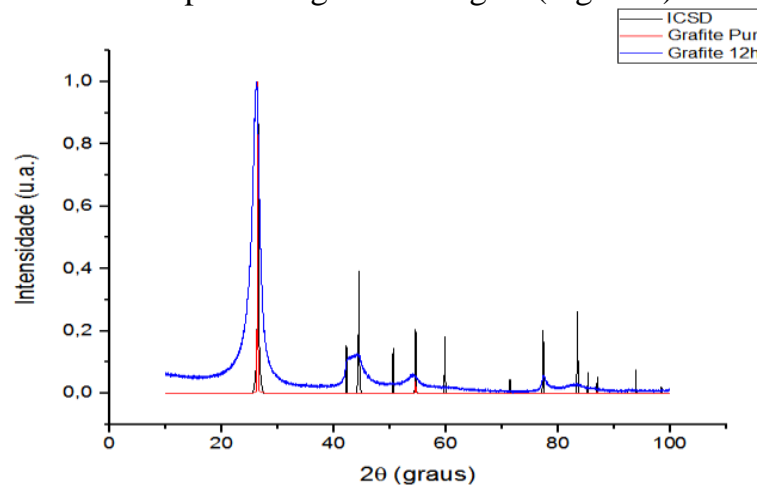

Figura 8 - Três parâmetros amostrais. Fonte: Autores, (2019).

Observa que o pico em aproximadamente $27^{\circ}$, referente ao conjunto de planos (002) do grafite, sofre alargamento após o processo de oxidação, bem como o aparecimento de um pico em aproximadamente $10^{\circ}$, atribuído ao conjunto de planos (002) do óxido de grafite, relacionado à expansão das folhas do grafite devido à inserção de grupos oxigenados. Essa relação de intensidade entre estes picos está associada ao grau de oxidação da amostra, ou seja, quanto maior é a intensidade do pico em $10^{\circ} \mathrm{em}$ relação a em $27^{\circ}$, mais oxidada é a amostra.

Os resultados apresentados neste relatório confirmaram a possibilidade de se realizar o estudo da grafite por análise comparativa utilizando a técnica de difração de raios $\mathrm{X}$, onde o controle dos parâmetros de síntese do óxido de grafite foi importante para a obtenção de um material com qualidade estrutural e o estudo do processo de moagem com $12 \mathrm{~h}$ da grafite se mostrou eficiente para a obtenção do óxido de grafeno a partir da do óxido de grafite.

\section{CONCLUSÃO}

A maioria das fontes de energias renováveis, com exceção da energia hidrelétrica, é de natureza intermitente e, portanto, precisam de sistemas de armazenamento. Os supercapacitores, entre os diversos sistemas de armazenamento, são os candidatos mais promissores para o armazenamento de energia não somente nas energias renováveis, como também em veículos híbridos e dispositivos portáteis, devido à sua elevada densidade de potência. Os eletrodos supercapacitores são quase sempre feitos de carbonos derivados da biomassa. Logo, este trabalho justifica-se pela relevância do tema, pois outro fator que está em alta nos últimos anos é a sustentabilidade empregada na biomassa residual amazônica. Após este estudo, pode-se aferir que a grafite se apresenta como uma estrutura do tipo lamelar, sendo formada por planos de átomos de carbono arranjados de forma hexagonal (camadas de grafeno) em hibridização do tipo sp2. Estas camadas são ligadas entre si, principalmente, por forças de interação fraca do tipo Van der Walls, que permite a intercalação de grandes quantidades de espécies químicas (moléculas e íons) no espaçamento entre os planos, uma vez que a distribuição eletrônica dos planos é facilmente rearranjada. Dessa forma, ao comparar os difratogramas das amostras de grafite puro com a grafite com 12 horas de moagem, por exemplo, verifica-se picos característicos relacionados com os parâmetros de rede e com o deslocamento 
atômico individual e da estrutura cristalina, podendo posteriormente realizar um estudo mais detalhado utilizando-se de outras ferramentas metodológicas, bem como a microscopia de varredura eletrônica. Assim, levando em consideração a questão econômica, social, tecnológica e ambiental, dado o reaproveitamento da biomassa residual de frutos amazônicos, tal como o caroço de açaí, um fruto consumido em grande escala pela região amazônica, o qual se apresenta muita das vezes sem destino correto após a extração de seu sumo, este trabalho torna-se plausível a fim de sintetizar o grafite por meio desse resíduo em escala nanotecnológica para diversas aplicabilidades, entre elas, a produção de supercapacitores.

\section{REFERÊNCIAS}

[1] Fernandes, D. A; Cardoso, A. C. D.; Almeida, L. M. L.; Kato, E. S. O Circuito Inferior da Economia Urbana na Amazônia: um estudo sobre o papel do mercado de batedores artesanais de açaí na economia da Região Metropolitana de Belém. In: Encontro Nacional da Associação Brasileira de Estudos Regionais e Urbanos, 13, 2015. Anais. Curitiba (PR), 2015.

[2] Turini, E. Conjuntura mensal: Açaí (fruto) - de 01 a 31/03/2015, Companhia Nacional de Abastecimento (CONAB).

[3] Pereira, S. C., Maehara L., Machado C. M. M., Farinas, C. S. 2015. 2G Ethanol from the whole sugarcane lignocellulosic biomass. Biotechnology for Biofuels, v. 8, p.1-16.

[4] Zhang L. L; Zhao, X. S. "Carbon-based materials as supercapacitor electrodes," Chemical Society Reviews, vol. 38, no. 9, pp. 2520-2531, 2009.

[5] Wang, H; Yuan, X; WU, Y. et al. Graphene-based materials: Fabrication, characterization and application for the decontamination of wastewater and wastegas and hydrogen storage/generation .Adv. Colloid Inter. Science, v. 195196, p. 19-40, 2013.

[6] Zhao, W.; Kido, G.; Hara, K.; Noguchi, H. Characterization of neutralized graphite oxide and its use in electric double layer capacitors. J. Electroanal. Chem. v 712, p. 185.

[7] Ferrero, G. A. Fuertes, A. B. and Sevilla, M. "From Soybean residue to advanced supercapacitors", Scientific Reports, vol. 5, Article ID 16618, 2015.

[8] Sekirifa, M. L; Hadj-Mahammed, S; Pallier, L; Baameur, D. "Preparation and characterization of an activated carbon from a date stones variety by physical activation with carbon dioxide," Journal of Analytical and Applied Pyrolysis, vol. 99, pp. 155-160, 2013.

[9] Sevilla, M; Mokaya. "Energy storage applications of activated carbons: supercapacitors and hydrogen storage," Energy \& Environmental Science, vol. 7, no. 4, pp. 1250-1280, 2014.

[10] Simons, P. L; Taberna, and F. Béguin, "Electrical double-layer capacitors and carbons for EDLCs," in Supercapacitors: Materials, Systems, and Applications, pp. 131-165, Wiley-VCH, 2013. 